\title{
Remarks on Infrared Dynamics in $\mathrm{QED}_{3}$
}

\author{
J. L. Boldo*, B. M. Pimentel ${ }^{\dagger}$ and J. L. Tomazelli ${ }^{\dagger \dagger}$, \\ * CBPF, Centro Brasileiro de Pesquisas Físicas \\ Rua Xavier Sigaud 150, 22290-180 Urca \\ Rio de Janeiro, Brazil \\ †IFT, Instituto de Física Teórica \\ Rua Pamplona 145, 01405-900 Bela Vista \\ São Paulo, Brazil \\ ${ }^{\dagger}$ DFQ - Faculdade de Engenharia, Universidade Estadual Paulista \\ Campus de Guaratinguetá, Av. Dr. Ariberto Pereira da Cunha, 333 \\ 12500-000 Guaratinguetá \\ São Paulo, Brazil \\ January 1, 2018
}

\begin{abstract}
In this work we study how the infrared sector of the interaction Hamiltonian can affect the construction of the $\mathrm{S}$ matrix operator of QED in $(2+1)$ dimensions.
\end{abstract}




\section{Introduction}

The issue of infrared divergences in quantum electrodynamics has been better understood since the discussion by Block and Nordsieck [1]. They pointed out the fact that the number of soft photons has no clear meaning and is not an observable. In fact, in any scattering process involving charged particles an infinite number of soft photons is emited collectively. Lately, Yennie, Frautsch and Suura 2] showed that finite cross sections from divergent S matrix elements are obtained if all radiative corrections are taken into account.

An alternative approach to the problem using coherent states to represent the asymptotic states with finite $\mathrm{S}$ matrix elements was proposed by Chung [3]. He showed that all the $\mathrm{S}$ matrix elements can be made finite if a suitable representation of photon states composed by coherent states is chosen, rather than the usual Fock representation. Kulish and Faddeev [4] proposed to modify not only the space of asymptotic states but also to redefine the scattering operator where the exact contribution of soft photons is taken into account [5, 6].

Interest in developing quantum electrodynamics in three dimensional space-time is still growing. Although some lower dimensional models have less physical content than those in four dimensions, they may provide new insights, by avoiding many problems encountered in four dimensions, serving as a theoretical laboratory. For instance, it is known that Einstein gravity in four dimensional spacetime suffers from serious problems such as its non-renormalizability ; however, the topological massive planar gravity is unitary and renormalizable. In the same way, the treatment of infrared divergencies in three dimensional gauge vector models can be performed by adding a topological gauge invariant mass term in the Lagrangean, which

provides an infrared cutoff to the theory [7, 8]. Furthermore, planar gauge theories have physical applications such as high-temperature limits of four dimensional field theories and the quantum Hall effect.

Here we are concerned with the investigation of the infrared behaviour of $\mathrm{QED}_{3}$, instead of calculating cross-sections explicitly. So we follow the pioneering work of Murota[9], which sheds light into the asymptotic limit of the theory. However, in contrast to the most of lower dimensional prob- 
lems which provide a simple setting where basic physical phenomena can be worked out, these divergencies are more drastic in three dimensions than in four and the interpretation of the results in the first case is not so obvious. The basic idea is to consider the exact contribution of the soft photons in the construction of the asymptotic time evolution operator in the S operator definition.

In the next section we shall see that the soft photon contribution to the $\mathrm{S}$ matrix may be isolated in a single time evolution operator, by making an arbitrary separation of the interaction Hamiltonian. Section 3 is devoted to an examination of the physical content of this operator. The conclusions are summarized in Section 4.

\section{Preliminaries}

The starting point of the approach is to perform an arbitrary splitting of the interaction Hamiltonian in $\mathrm{H}_{s}$, which contains soft photons, and $\mathrm{H}_{h}$ the remaining one, i.e.,

$$
H(t)=H_{s}(t)+H_{h}(t) .
$$

Thus, the $\mathrm{S}$ matrix is given by

$$
\begin{aligned}
S & =T \exp \left[-i \int_{-\infty}^{+\infty} d t\left(H_{s}(t)+H_{h}(t)\right)\right] \\
& =U\left(\infty, t_{0}\right) S_{h} U\left(t_{0},-\infty\right)
\end{aligned}
$$

where

$$
U\left(t, t_{0}\right)=T \exp \left[-i \int_{t_{0}}^{t} d \tau H_{s}(\tau)\right]
$$

and

$$
S_{h}=T \exp \left[-i \int_{-\infty}^{+\infty} d t H_{h}(t)\right]
$$

Note that the soft part of the Hamiltonian is all contained in the operator $U\left(t, t_{0}\right)$, so that its analysis will be the main purpose of this paper. In addition, equation (2.1) is of fundamental importance because one stablishes the relation between the infrared (IR) behaviour and asymptotic dynamics. 
In order to prove this relation we use the fact that $H_{s}(t)$ and $H_{h}(t)$ commute. Therefore,

$$
\begin{aligned}
S= & T\left\{\operatorname { e x p } [ - i \int _ { - \infty } ^ { + \infty } d t H _ { s } ( t ) ] \left[1-i \int d t_{1} H_{h}\left(t_{1}\right)+\right.\right. \\
& \left.\left.+\frac{(-i)^{2}}{2 !} \int d t_{1} \int d t_{2} H_{h}\left(t_{1}\right) H_{h}\left(t_{2}\right)+\ldots\right]\right\} \\
= & T\left\{U(+\infty,-\infty)-i \int d t_{1} U\left(+\infty, t_{1}\right) H_{h}\left(t_{1}\right) U\left(t_{1},-\infty\right)\right. \\
& \left.+\frac{(-i)^{2}}{2 !} \int . d t_{1} \int d t_{2} U\left(+\infty, t_{1}\right) H_{h}\left(t_{1}\right) U\left(t_{1}, t_{2}\right) H_{h}\left(t_{2}\right) U\left(t_{2},-\infty\right)+\ldots\right\} .
\end{aligned}
$$

In this way, the $\mathrm{n}^{\text {th }}$ order term of $S$ matrix in the perturbation series with respect to $H_{h}(t)$ is given by

$$
\begin{aligned}
S^{(n)}= & (-i)^{n} \int d t_{1} \ldots d t_{n} \theta\left(t_{1}-t_{2}\right) \theta\left(t_{2}-t_{3}\right) \ldots \theta\left(t_{n-1}-t_{n}\right) \\
& \times U\left(+\infty, t_{1}\right) H_{h}\left(t_{1}\right) U\left(t_{1}, t_{2}\right) H_{h}\left(t_{2}\right) \ldots U\left(t_{n-1}, t_{n}\right) H_{h}\left(t_{n}\right) U\left(t_{n},+\infty\right) .
\end{aligned}
$$

Inserting complete sets before and after $H_{h}\left(t_{i}\right)$, we see that the dependence of $H_{h}\left(t_{i}\right)$ is given by the difference between energies of states before and after $t_{i}$

$$
H_{h}\left(t_{i}\right) \sim e^{i \Delta E t}
$$

On the other hand, we shall see that the $t_{i}$ dependence in the operators $U\left(t_{n-i}, t_{i}\right)$ and $U\left(t_{i}, t_{n+i}\right)$ is as follows

$$
U\left(t_{n-i}, t_{i}\right), U\left(t_{i}, t_{n+i}\right) \sim \sum e^{i \sum \frac{p \cdot k}{E} t_{i}} .
$$

But, taking into account that the energy of soft photons is very small that the hard ones, namely,

$$
\left|\Delta E_{i}\right| \gg\left|\sum \frac{p \cdot k}{E_{i}}\right|,
$$

we may use the approximation 


$$
U\left(t_{n-i}, t_{i}\right) H_{h}\left(t_{i}\right) U\left(t_{i}, t_{n+i}\right) \simeq U\left(t_{n-i}, t_{0}\right) H_{h}\left(t_{i}\right) U\left(t_{0}, t_{n+i}\right) .
$$

Since $U\left(t_{i}, t_{0}\right) \simeq 1$, (2.5) can be approximated by

$$
S^{(n)}=U\left(\infty, t_{0}\right) \frac{(-i)^{n}}{n !} \int d t_{1} \ldots d t_{n} T\left[H_{h}\left(t_{1}\right) \ldots H_{h}\left(t_{n}\right)\right] U\left(t_{0},-\infty\right) .
$$

The above result holds for all $\mathrm{n}$, and hence (2.1) is proved.

\section{Construction of the Infrared Operator in $\mathrm{QED}_{3}$}

We now shall construct $U\left(t, t_{0}\right)$ which contains the infrared behaviour of the theory.

In the interaction picture, the QED Hamiltonian of the system of charged particles and photons is

$$
H(t)=\int d \vec{x} \Psi(x) \gamma_{\mu} \bar{\Psi}(x) \cdot A^{\mu}(x),
$$

where $\Psi$ and $\bar{\Psi}$ are the operators of the electron-positron field and $A^{\mu}$ the operator of the electromagnetic gauge field. These field operators can be expanded in terms of the creation and annihilation operators as follows:

$$
\begin{gathered}
A_{\mu}(x)=\frac{1}{2 \pi} \int \frac{d \vec{k}}{\sqrt{2 \omega_{k}}}\left(a_{\mu}(\vec{k}) e^{-i k \cdot x}+a_{\mu}^{\dagger}(\vec{k}) e^{i k \cdot x}\right) \\
\Psi(x)=\Psi^{(+)}(x)+\Psi^{(-)}(x) \\
\bar{\Psi}(x)=\bar{\Psi}^{(+)}(x)+\bar{\Psi}^{(-)}(x)
\end{gathered}
$$

where

$$
\Psi^{(+)}(x)=\frac{1}{2 \pi} \int d \vec{p} \sqrt{\frac{m}{p_{0}}} \sum_{r} b_{r}(\vec{p}) u_{r}(\vec{p}) e^{-i p \cdot x}
$$




$$
\begin{aligned}
& \Psi^{(-)}(x)=\frac{1}{2 \pi} \int d \vec{p} \sqrt{\frac{m}{p_{0}}} \sum_{r} d_{r}^{\dagger}(\vec{p}) v_{r}(\vec{p}) e^{i p \cdot x} \\
& \bar{\Psi}^{(-)}(x)=\frac{1}{2 \pi} \int d \vec{p} \sqrt{\frac{m}{p_{0}}} \sum_{r} b_{r}^{\dagger}(\vec{p}) \bar{u}_{r}(\vec{p}) e^{i p \cdot x} \\
& \bar{\Psi}^{(+)}(x)=\frac{1}{2 \pi} \int d \vec{p} \sqrt{\frac{m}{p_{0}}} \sum_{r} d_{r}(\vec{p}) \bar{v}_{r}(\vec{p}) e^{-i p \cdot x}
\end{aligned}
$$

Here $a_{\mu}(\vec{k}), b_{r}(\vec{p})$ and $d_{r}(\vec{p})$, are the annihilation operators of photon, electron and positron respectively. $u_{r}(\vec{p})$ and $v_{r}(\vec{p})$ are the corresponding spinor amplitudes. Inserting these quantities into (3.9), one obtains for $H(t)$ an integral over the momenta $\vec{p}, \vec{q}$ and $\vec{k}$ of the fermions and photons, which are related by the constraint

$$
\vec{p}+\vec{q}=\vec{k}
$$

We now turn to investigate the infrared behaviour of the interaction Hamiltonian, by making use of the following asymptotic approximations

$$
\begin{gathered}
p^{0}-q^{0} \mp \omega \simeq \mp \frac{p \cdot k}{p^{0}} . \\
p^{0}+q^{0} \pm \omega \simeq 2 p^{0}
\end{gathered}
$$

and

$$
\begin{aligned}
\bar{u}_{r}(p) \gamma^{\mu} u_{s}(q) & =\frac{1}{2 m} \bar{u}_{r}(p)\left[p^{\mu}+q^{\nu}+i \sigma^{\mu \nu}\left(p_{\nu}-q_{\nu}\right)\right] u_{s}(q) \\
& \simeq \frac{p^{\mu}}{m} \delta_{r s},
\end{aligned}
$$

It is easy to see that the time dependence of $H_{s}(t)$ split into two groups, one is proportional to $e^{ \pm 2 i p_{o} t}$ and the other to $e^{ \pm i \frac{k \cdot p}{p^{0}} t}$. Thus, after time integration, the former is smaller than the latter due the fact that 


$$
\left|p_{0}\right| \gg\left|\frac{p \cdot k}{p_{0}}\right|
$$

so that all those terms of the first group can be neglected.

It is important to notice that the vertex approximation (3.14) is spin independent; therefore, it is usually called eikonal in a sense that charged particles act as pointlike sources.

Replacing these approximations into (3.9) one finally obtains the interaction Hamiltonian, which describes the interaction between charged particles and soft photons:

$$
H_{s}(t)=\frac{1}{(2 \pi)} \int d \vec{k} \int d \vec{p} \frac{1}{\sqrt{2 \omega}} \frac{p^{\mu}}{p_{0}} \rho(\vec{p})\left[a_{\mu}(\vec{k}) e^{-i \frac{k \cdot p}{p^{0}} t}+a_{\mu}^{\dagger}(\vec{k}) e^{i \frac{k \cdot p}{p^{0}} t}\right]
$$

where $\rho(\vec{p})$ is the charge density operator

$$
\rho(\vec{p})=e\left[b_{r}^{\dagger}(\vec{p}) b_{r}(\vec{p})-d_{r}^{\dagger}(\vec{p}) d_{r}(\vec{p})\right] .
$$

Substituting (3.15) in (2.2) we obtain $U\left(t, t_{0}\right)$ in form of a T-ordered product. This expression can be transformated in to an ordinary product by noting that $H_{s}(t)$ possesses the following property

$$
\left[H_{s}\left(t_{1}\right), H_{s}\left(t_{2}\right)\right]=c-\text { number }, \forall t_{1} \neq t_{2},
$$

which commutes with $H_{s}(t)$ for all $t, t_{1}, t_{2}$. By making use of this commutation relation, one readily obtain a more suitable form for the time evolution operator

$$
U\left(t, t_{0}\right)=\exp \left\{R\left(t, t_{0}\right)\right\} \exp \left\{i \phi\left(t, t_{0}\right)\right\},
$$

where

$$
R\left(t, t_{0}\right)=\exp \left[-i \int_{t_{0}}^{t} d \tau H_{s}(\tau)\right]
$$

and

$$
i \phi\left(t, t_{0}\right)=\exp \left\{-\frac{1}{2} \int_{t_{0}}^{t} d \tau \int_{t_{0}}^{\tau} d s\left[H_{s}(\tau), H_{s}(s)\right]\right\} .
$$

The $S$ matrix is defined in (2.1) in the limit $t, s \rightarrow \pm \infty$. In order to simplify the calculations we shall take $t_{0}=0$, since the time $t_{0}$ is a finite parameter chosen between $-\infty<t_{0}<+\infty$. 
Then, the construction of the asymptotic part of the $S$ matrix is made by replacing these limits in the operators $U\left(t, t_{0}\right)$, namely,

$$
U( \pm \infty, 0)=\exp [R( \pm \infty, 0)] \exp [i \phi( \pm \infty, 0)]
$$

where

$$
R( \pm \infty, 0)=\frac{1}{(2 \pi)} \int \frac{d \vec{k}}{\sqrt{2 \omega}} \int d \vec{p} \frac{p^{\mu}}{p \cdot k} \rho(\vec{p})\left[a_{\mu}(\vec{k})-a_{\mu}^{\dagger}(\vec{k})\right]
$$

and

$$
\phi( \pm \infty, 0)=\mp \frac{i}{4 \pi} \int d \vec{p} \int d \vec{q} \hat{p} \cdot \widehat{q} \rho(\vec{p}) \rho(\vec{q}) \int \frac{d \vec{k}}{2 \omega} \frac{\delta[k \cdot(\widehat{p}-\widehat{q})]}{(\widehat{q} \cdot k)}
$$

Here

$$
\widehat{p} \equiv \frac{p}{p_{0}}, \widehat{q} \equiv \frac{q}{q_{0}}
$$

The infrared behaviour of the phase operator can be most easily deter-

mined in the nonrelativistic limit, $|\vec{v}| \ll c$. In this case, it can be rewritten as

$$
\phi( \pm \infty, 0)= \pm \frac{i}{4 \pi} \int d \vec{p} \int d q \rho(\vec{p}) v_{p q} \rho(\vec{q})\left(-\frac{1}{\omega}\right)_{\lambda}^{\Delta},
$$

where the cutoff parameter $\Delta$ was used to define the infrared sector and $\lambda \rightarrow 0$. Also,

$$
v_{p q} \equiv \frac{p \cdot q}{\sqrt{(p \cdot q)^{2}-p^{2} q^{2}}}
$$

is usually called phase operator.

\section{Discussion}

From the above result, we see that the I.R. structure of the theory is made explicit. Furthermore, it is instructive to compare the I.R. behaviour of the phase operator (3.24) in $3 \mathrm{D}$ and that one in $4 \mathrm{D}$. In the latter case, the divergent Coulomb phase as a function of the photon frequency does have 
the same logarithm behaviour as that one when the same infrared analysis is made in terms of the asymptotic times [9, 10], while it is not true in 3D[11].

Moreover, the operators $R( \pm \infty, 0)$ are not well defined in the Fock space for photons and charged particles. In fact, when applied on states which belong to the Fock space, they yield coherent states. In other words, they force us to introduce a new space of asymptotic states, the space of coherent states, which contains a infinite number of soft quanta and, therefore, becomes necessary to sum over final and initial states and not only over final states as claimed by the Block-Nordsieck theory.

\section{Acknowledgements}

J.L.B. thanks $\mathrm{CNP} q$ for financial support; B.M.P. and J.L.T. are partially supported by CNPq.

\section{References}

[1] F. Bloch and A. Nordsieck, Phys. Rev. 52, 54, (1937);

[2] D. R. Yennie, S.C. Frautschi, and H. Suura, Ann. Phys. (New York), 13, 379 (1961);

[3] V. Chung, Phys. Rev. 140B, 1100 (1965);

[4] P. Kulish and L. Faddeev, Teor. Mat. Fiz. 4, 153 (1970) [Theor. Math. Phys. 4, 745 (1970)];

[5] T. W. B. Kibble, Phys. Rev., 173, 1527; 173, 1882; 175, 1624 (1968);

[6] D. Zwanziger, Phys. Rev. D11, 3481, 3504 (1975);

[7] S. Deser, R. Jackiw, and A. Templeton, Ann. Phys. 140, 372 (1982); Phys. Rev. Lett. 48, 975 (1982);

[8] S. Deser and Z. Yang, Class. Quantum Grav. 7, 1603 (1990);

[9] T. Murota, Prog. Theor. Phys. 24, 1109, (1960); 
[10] M. Ciafaloni, Phys. Lett. 150B, 379 (1985); S. Catani, M. Ciafaloni and G. Marchesini, Nucl. Phys. B264, 588 (1986); S. Catani, M. Ciafaloni, Nucl. Phys. B289, 535 (1987);

[11] J. L. Boldo, B. M. Pimentel and J. L. Tomazelli, Can. J. Phys. 76, 69 (1998). 